\title{
Corrigendum: Occurrence bilateral bronchospasm consequently after unilateral bronchospasm (Korean J Anesthesiol 2013 December 65(6 Suppl): S28-S29)
}

\author{
Yun Hee Kim, Woon Young Kim, Joong Il Kim, Doo Jae Min, and \\ Yoon Sook Lee \\ Department of Anesthesiology and Pain Medicine, Korea University Ansan Hospital, Ansan, Korea
}

\begin{abstract}
The article by Kim YH, et al. entitled, "Occurrence bilateral bronchospasm consequently after unilateral bronchospasm” (Korean J Anesthesiol 2013 December 65(6 Suppl): S28-S29) contained an error in author’s name.
\end{abstract}

Korean J Anesthesiol 2013 December 65(6 Suppl): S28-S29

Before correction:

Yun Hee Kim, Woon Young Kim, Joong Il Kim, Doo Jae Min, and Yoon Sook Lee

The correct information is found below:

Yun Hee Kim, Woon Young Kim, Joong Il Kim, Too Jae Min, and Yoon Sook Lee

The fourth author's name was misspelled as Doo Jae Min. The correct spelling is Too Jae Min.

The authors apologize for any inconvenience this mistake may have caused. 